\title{
Non-Conforming Finite Element Methods for Nonmatching Grids in Three Dimensions
}

\author{
Wayne McGee and Padmanabhan Seshaiyer* \\ Texas Tech University, Mathematics and Statistics (padhu@math.ttu.edu)
}

\begin{abstract}
Summary. In the last decade, non-conforming domain decomposition methods such as the mortar finite element method have been shown to be reliable techniques for several engineering applications that often employ complex finite element design. With this technique, one can conveniently assemble local subcomponents into a global domain without matching the finite element nodes of each subcomponent at the common interface. In this work, we present computational results for the convergence of a mortar finite element technique in three dimensions for a model problem. We employ the mortar finite element formulation in conjunction with higher-order elements, where both mesh refinement and degree enhancement are combined to increase accuracy. Our numerical results demonstrate optimality for the resulting non-conforming method for various discretizations.
\end{abstract}

\section{Introduction}

As computational resources are rapidly increasing, numerical modeling of physical processes is being performed on increasingly complex domains. Often an analysis may be performed by decomposing the global domain into several local subdomains, each of which can be modeled independently. The global domain can then be reconstructed by assembling the subdomains appropriately. In the standard conforming method, it is required that the corners of a given element intersect other elements only on their corners, that is, corners must not coincide with edges of other elements. It is often infeasible or inconvenient to coordinate the decomposition and reassembly processes so that the subdomains conform at the common interfaces. The use of a nonconforming method circumvents this difficulty. In practical applications, the non-conforming method has two noteworthy advantages. First, the discretization of the domain can be selectively increased in localized regions, such as around corners or other features where the error in the solution is likely to be

\footnotetext{
* This work of this author is supported in part by the National Science Foundation under Grant DMS 0207327.
} 
greatest. This allows for greater accuracy in the method without the computational load associated with increasing the discretization of the entire domain.

Another practical benefit of the non-conforming method is that the process may be utilized to connect independently analyzed substructures in a large problem. For example, in the construction of an aircraft, the fuselage and wing structures may have been analyzed independently by different engineers, possibly in different organizations. It is highly unlikely that the independently constructed meshes of each subcomponent would coincide when assembled. Moreover, transition meshing could become highly complex and expensive to achieve. The non-conforming approach eliminates this need.

The mortar finite element method (Bernardi et al. [1993], Belgacem [1999], Seshaiyer and Suri [2000b], D. Braess and Wieners [2000], Wohlmuth [2000] and references therein) is an example of a non-conforming technique. In the last decade, there has been a lot of research on the theoretical and computational aspects of this domain decomposition technique (Seshaiyer and Suri [1998], Seshaiyer [2003], Ewing et al. [2000], Braess et al. [1999]). It has been well-established that the mortar finite element method yields optimal results both in the presence of highly non-quasiuniform meshes and high polynomial degree (Seshaiyer and Suri [2000a]) and also preserves the optimal rates afforded by conforming $h, p$, and $h p$ discretizations for a variety of applications (Belgacem et al. [2000, 2003]).

In the last few years, the extension of the mortar finite element technique has been analyzed (see Belgacem and Maday [1997], Braess and Dahmen [1998], Kim et al. [2001] and references therein). In Belgacem and Maday [1997] the mortar finite element method was extended for the special case of linear polynomials. However, the method is difficult to generalize for a general mesh of parallelograms for any polynomial degree. To circumvent this, a variant of the mortar method, $M_{1}$, was introduced in (Seshaiyer and Suri [2000a], Seshaiyer [2003]) which easily extends the technique to any number of dimensions. The computational performance of this method, however, was not tested which is the focus, herein. In this paper, we computationally validate the convergence behavior for the mortar finite element formulation for a timedependent model problem in three-dimensions. In particular, we show via numerical experiments that the $M_{1}$ mortar method is stable and behaves as well as the conforming finite element method.

\section{Model problem and its discretization}

Consider the model problem for $\mathbf{x}=\left(x_{1}, x_{2}, x_{3}\right) \in \Omega, t>0$ :

$$
\frac{\partial u(\mathbf{x}, t)}{\partial t}-\nabla \cdot(P(\mathbf{x}) \nabla u(\mathbf{x}, t))+Q(\mathbf{x}) u(\mathbf{x}, t)=f(\mathbf{x}, t),
$$

where $P$ is uniformly positive and $Q$ is a nonnegative function in the bounded domain $\Omega$, with the boundary and initial conditions 


$$
\begin{aligned}
u(\mathbf{x}, t) & =0 \quad \text { for } \mathbf{x} \in \partial \Omega, \\
u(\mathbf{x}, 0) & =g(\mathbf{x}) .
\end{aligned}
$$

Discretizing time using a backward Euler scheme yields

$$
-\nabla \cdot\left(P_{(0)} \nabla u_{n}\right)+Q_{(0)} u_{n}=f_{n(0)},
$$

where $P_{(0)}=(\Delta t) P, \quad Q_{(0)}=1+(\Delta t) Q, \quad$ and $f_{n(0)}=u_{n-1}+(\Delta t) f_{n}$.

Let us for simplicity, decompose $\Omega$ into two geometrically conforming nonoverlapping subdomains $\Omega_{1}$ and $\Omega_{2}$, which share a common interface $\Gamma$ (denoted by the dotted line). For each subdomain $\Omega_{i}$, we consider a regular sequence of geometrically conforming triangulations $\tau_{i}$. Note that, no compatibility is assumed between meshes in different subdomains, i.e. the meshes of $\Omega_{1}$ and $\Omega_{2}$ need not match on $\Gamma$. This is illustrated in the figure 1 .
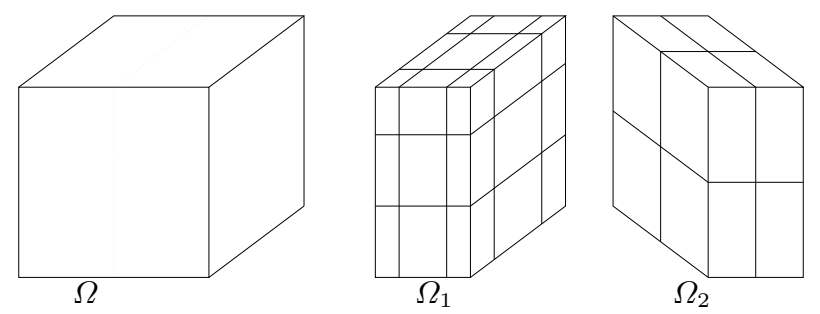

Fig. 1. Geometrically conforming decomposition of $\Omega$ partitioned into $\Omega_{1}$ and $\Omega_{2}$ with non-conforming meshes

Let $u_{n}^{(i)}$ denote the interior solution in each $\Omega_{i}$, which satisfies the global continuity restriction $u_{n}^{(1)}(\mathbf{x})=u_{n}^{(2)}(\mathbf{x})$ for $\mathbf{x} \in \Gamma$. Due to the non-conformity of the grids across $\Gamma$, we enforce this continuity in a weak sense as

$$
b\left(u_{n}, \psi\right):=\int_{\Gamma}\left(u_{n}^{(1)}-u_{n}^{(2)}\right) \psi d x=0 \quad \forall \psi \in H^{-1 / 2}(\Gamma) .
$$

Let us now describe the weak formulation of our model problem (4) as a mixed method formulation, which is a convenient method for implementation. Using standard Sobolev space notation, define $H_{D}^{1}\left(\Omega_{i}\right)=\left\{v \in H^{1}\left(\Omega_{i}\right) \mid v=\right.$ 0 on $\left.\partial \Omega_{i} \bigcap \partial \Omega_{D}\right\}$. The weak form of (4) then becomes: For $i=1,2$, find $u_{n}^{(i)} \in H_{D}^{1}\left(\Omega_{i}\right)$ such that for all $v_{i} \in H_{D}^{1}\left(\Omega_{i}\right)$,

$\int_{\Omega_{i}} P_{(0)} \nabla u_{n}^{(i)} \cdot \nabla v_{i} d x-\int_{\Gamma} P_{(0)} \frac{\partial u_{n}^{(i)}}{\partial n} v_{i} d s+\int_{\Omega_{i}} Q_{(0)} u_{n}^{(i)} v_{i} d x=\int_{\Omega_{i}} f_{n(0)} v_{i} d x$

Let $\lambda=-P_{(0)} \frac{\partial u_{n}^{(1)}}{\partial n}=P_{(0)} \frac{\partial u_{n}^{(2)}}{\partial n}$. Define the spaces $\tilde{V}=\left\{v \in L^{2}(\Omega),\left.v\right|_{\Omega_{i}} \in\right.$ $\left.H_{D}^{1}\left(\Omega_{i}\right)\right\}$ and $\Lambda=\left\{\psi \in \mathcal{D}^{\prime}(\Gamma),\left.\psi\right|_{\Gamma} \in H^{-\frac{1}{2}}(\Gamma)\right\}$ (where $\mathcal{D}^{\prime}$ is the Schwarz set of distributions) equipped with their respective norms. 
For each $T \in \tau_{i}$, denote the set $S_{p}(T)$ to be all polynomials generated by the serendipity (or trunk) space families. Hence $S_{2}(T)$ has 20 degrees of freedom. We have used these spaces for our computations in the next section. Assume the finite element spaces $V_{F}^{(i)}=\left\{u \in H^{1}\left(\Omega_{i}\right)|u|_{T} \in S_{p}(T), u=\right.$ 0 on $\left.\partial \Omega_{i} \cap \partial \Omega_{D}\right\}$ are given. We can then define the non-conforming space $\tilde{V}_{F}=\left\{u \in L_{2}(\Omega)|u|_{\Omega_{i}} \in V_{F}^{(i)}\right\} \subset \tilde{V}$.

To define the finite-dimensional Lagrange multiplier space, let us suppose that the mesh on the interface $\Gamma$ matches the mesh on $\Omega_{1}$ (Note that this choice is arbitrary). For $K \subset \mathbb{R}^{2}$, we denote by $Q_{p, s}(K)$ the set of polynomials on $K$ which is of degree $p$ in $x$ and $s$ in $y$ (so that $\left.Q_{p, p}(K)=Q_{p}(K)\right)$. Let us denote the rectangles in the mesh on the interface $\Gamma$ by $K_{i j}, 0 \leq i, j \leq N$. Then the Lagrange multiplier space will be defined as $\Lambda_{F}=\left\{\chi \in C(\Gamma): \chi_{\left.\right|_{K_{i j}}} \in Q_{p-1}\left(K_{i j}\right)\right\} \subset \Lambda$ (see Figure 2). The

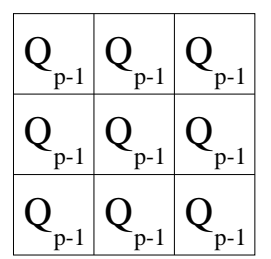

Fig. 2. Lagrange multiplier space for $M_{1}$ method

associated mortar method is called the $M_{1}$ mortar finite element method, and has been implemented in the next section. It can be shown that this choice of the Lagrange multiplier space leads to optimal results in three-dimensions by extending the arguments of Seshaiyer and Suri [2000a] and Seshaiyer [2003].

Let us now define $\stackrel{0}{\mathrm{~V}}_{F}^{\Gamma}=\left\{\left.u\right|_{\Gamma}, u \in V_{F}^{(i)}\right\} \cap H_{D}^{1}(\Gamma)$. Then for any $z \in L^{2}(\Gamma)$, we define the space $X_{F}^{\Gamma}(z)=\left\{w \in \stackrel{0}{\mathrm{~V}}_{F}^{\Gamma}, \int_{\Gamma}(w-z) \chi d s=0 \forall \chi \in \Lambda_{F}\right\}$. Let us now make the following restriction.

Condition I: $X_{F}^{\Gamma}(z) \neq \emptyset$ for all $z \in L_{2}(\Gamma)$.

If Condition I holds, then one can prove that the mixed formulation satisfies the inf-sup condition:

$$
\inf _{\substack{\lambda \in \Lambda_{F} \\ \lambda \neq 0}} \sup _{\substack{v \in \tilde{V}_{F}\\}} \frac{b(v, \lambda)}{\|v\|_{\tilde{V}}\|\lambda\|_{\Lambda}}>0
$$

Let the finite dimensional spaces $V_{F}^{(i)}$ and $\Lambda_{F}$ be spanned by basis functions $\left\{\Psi_{j}^{(i)}\right\}_{j=1}^{N_{i}}$ and $\left\{\Phi_{j}\right\}_{j=1}^{N_{\lambda}}$ respectively. Writing $u_{n}^{(i)}=\sum_{k=1}^{N_{i}} a_{k}^{(i)} \Psi_{k}^{(i)}$ and $\lambda=\sum_{k=1}^{N_{\lambda}} b_{k} \Phi_{k}$ respectively, (6) and (5) yield a discrete system of integral equations, which can be written in block matrix form as: 


$$
\left[\begin{array}{ccc}
A_{1} & 0 & B_{1} \\
0 & A_{2} & B_{2} \\
B_{1}^{T} & B_{2}^{T} & 0
\end{array}\right]\left[\begin{array}{c}
\mathbf{a}^{(\mathbf{1})} \\
\mathbf{a}^{(2)} \\
\mathbf{b}
\end{array}\right]=\left[\begin{array}{c}
\mathbf{F}_{\mathbf{1}} \\
\mathbf{F}_{\mathbf{2}} \\
0
\end{array}\right]
$$

Here $\mathbf{a}^{(\mathbf{i})}=\left\{a_{1}^{(i)}, a_{2}^{(i)}, \ldots, a_{N_{i}}^{(i)}\right\}, \mathbf{b}=\left\{b_{1}, b_{2}, \ldots, b_{N_{\lambda}}\right\}$ and

$$
\begin{gathered}
A_{i(s j)}=\int_{\Omega_{i}} P_{(0)}^{(i)} \nabla \Psi_{s}^{(i)} \cdot \nabla \Psi_{j}^{(i)} d x+\int_{\Omega_{i}} Q_{(0)}^{(i)} \Psi_{s}^{(i)} \Psi_{j}^{(i)} d x \\
B_{1(j s)}=\int_{\Gamma} \Phi_{s} \Psi_{j}^{(1)} d s \quad B_{2(j s)}=-\int_{\Gamma} \Phi_{s} \Psi_{j}^{(2)} d s \quad F_{i(j)}=\int_{\Omega_{i}} f_{n(0)}^{(i)} \Psi_{j}^{(i)} d x
\end{gathered}
$$

for $i=1,2$. Note that the invertibility of the stiffness matrix in (7) is related to Condition I.

\section{Numerical Results}

In this section, we demonstrate the performance of the numerical technique described. Our computations were performed for the model problem (1) on the domain $\Omega=(-1,1) \times(-1,1) \times(-1,1)$, and we decompose this domain into $\Omega_{1}=(-1,1) \times(-1,0) \times(-1,1)$ and $\Omega_{2}=(-1,1) \times(0,1) \times(-1,1)$. We take $h_{1}$ subintervals along the $x, y$, and $z$ axes for $\Omega_{1}$, and $h_{2}$ subintervals for $\Omega_{2}$. A sample partition of $\Omega$ into two subdomains with $h_{1}=3$ and $h_{2}=2$ is shown in Figure 1. Note that the grids do not match on the interface. For our experiments, we consider uniform polynomial degrees $p$ in both subdomains.

\section{Steady-state, constant coefficients}

Our initial experiment involves a steady-state $\left(\frac{\partial u}{\partial t}=0\right)$ equation with constant coefficients $P=Q=1$. We choose the right hand side $f$ such that our exact solution is $u(x, y, z)=\left(1-x^{2}\right)\left(1-y^{2}\right)\left(1-z^{2}\right)$.

We consider the $h$-version for the non-conforming method for the combinations $\left(h_{1}, h_{2}\right)=\{(3,3),(3,4),(4,4), \ldots,(7,8),(8,8)\}$ with polynomial degrees $p=1$ and $p=2$. The results are demonstrated for both the $L_{2}$ (Figure $3(\mathrm{a})$ ) and $H^{1}$ (Figure $3(\mathrm{~b})$ ) errors. For our computations, we have used tensor products of one-dimensional Gauss-Legendre quadratures for numerical integration and the errors have been computed at the Gauss points on the rectangular grids. Due to our simplified geometry with a smooth regular solution, we not only get optimal solutions but one can also observe superconvergence rates. Although not obvious, one may need to perform a detailed analysis for the mixed mortar method, to study this superconvergence behaviour following the details Ewing and Lazarov [1993].

Figure 4 demonstrates the performance of the non-conforming method versus the conforming method for $p=2$. For this experiment, the $L_{2}$ error for 

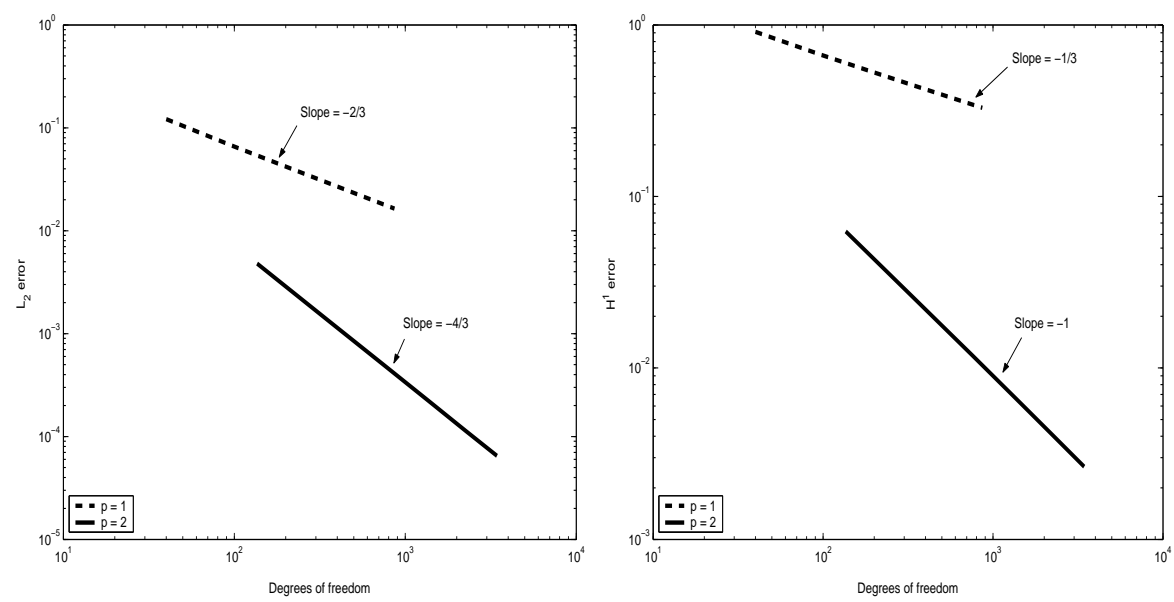

Fig. 3. Steady-state convergence: (a) $L^{2}$ error, (b) $H^{1}$ error

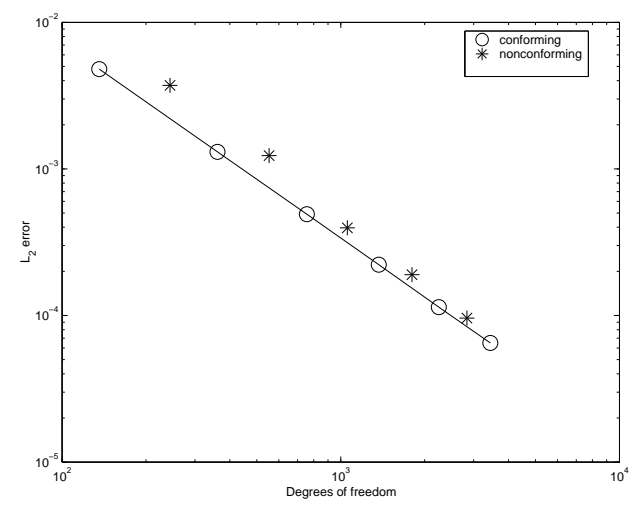

Fig. 4. Conforming versus non-conforming method

the conforming meshes $\left(h_{1}, h_{2}\right)=\{(3,3),(4,4), \ldots,(8,8)\}$ (circles) is plotted against the non-conforming meshes $\left(h_{1}, h_{2}\right)=\{(3,4),(4,5), \ldots,(7,8)\}$ (asterisks). The results indicate that the non-conforming method performs no worse than the conforming method in higher dimensions.

\section{Steady-state, varying coefficients}

Next, we performed computations for the steady-state problem with $P(y)=$ $\sin y+2$ and $Q(y)=\cos y+2$. The results of this experiment are shown in Table 1 . We denote by DOF the number of degrees of freedom, which is the size of the stiffness matrix in (7). $L_{2}$ and $H^{1}$ denote the errors in the respective norms, and $L_{2} \%$ and $H^{1 \%}$ are the respective relative errors. 
Table 1. Nonconstant coefficients, $p=2$

\begin{tabular}{lllllll}
\hline$h_{1}$ & $h_{2}$ & DOF & $L_{2}$ & $L_{2} \%$ & $H^{1}$ & $H^{1} \%$ \\
\hline 3 & 3 & 136 & 0.004798 & 0.435505 & 0.062567 & 1.948016 \\
3 & 4 & 244 & 0.003711 & 0.336868 & 0.048629 & 1.514050 \\
4 & 4 & 361 & 0.001307 & 0.118605 & 0.024271 & 0.755660 \\
4 & 5 & 553 & 0.001238 & 0.112389 & 0.020820 & 0.648217 \\
5 & 5 & 756 & 0.000491 & 0.044614 & 0.011831 & 0.368343 \\
5 & 6 & 1056 & 0.000395 & 0.035888 & 0.009703 & 0.302095 \\
6 & 6 & 1369 & 0.000222 & 0.020174 & 0.006605 & 0.205649 \\
6 & 7 & 1801 & 0.000190 & 0.017269 & 0.005602 & 0.174420 \\
7 & 7 & 2248 & 0.000114 & 0.010382 & 0.004051 & 0.126116 \\
7 & 8 & 2836 & 0.000096 & 0.008694 & 0.003458 & 0.107656 \\
8 & 8 & 3441 & 0.000065 & 0.005862 & 0.002658 & 0.082762 \\
\hline
\end{tabular}

Table 2. Convergence in $\Delta t$

\begin{tabular}{c|lrr|lr|rrr}
\hline $\mathrm{n}$ & $L_{2}$ & $8: 8$ & $H^{1}$ & $L_{2}$ & $7: 8$ & $H^{1}$ & $L_{2}$ & $7: 7$ \\
\hline 2 & 0.550825 & 1.605919 & 0.550899 & 1.606458 & 0.554554 & 1.629562 \\
4 & 0.435470 & 0.802964 & 0.275490 & 0.803821 & 0.279264 & 0.838348 \\
8 & 0.137706 & 0.401490 & 0.137790 & 0.402982 & 0.141801 & 0.458332 \\
16 & 0.068853 & 0.200759 & 0.068948 & 0.203510 & 0.073412 & 0.290824 \\
32 & 0.034427 & 0.100407 & 0.034545 & 0.105592 & 0.039823 & 0.228157 \\
64 & 0.017214 & 0.050257 & 0.017377 & 0.059764 & 0.023938 & 0.208207 \\
\hline
\end{tabular}

\section{Time-dependent case}

Our final experiment confirmed convergence for the unsteady equation. The exact solution was chosen to be $u(x, y, z, t)=t\left(1-x^{2}\right)\left(1-y^{2}\right)\left(1-z^{2}\right)$. We considered several matching and non-matching mesh combinations and the results for the combinations $\left(h_{1}, h_{2}\right)=\{(8,8),(7,8),(7,7)\}$ for polynomial degree 2, are presented in Table 2. Our computations were run from time $t=0$ to time $t=1$, with varying numbers of time steps $n$. The results not only demonstrate convergence as we refine the time discretization but also suggest that the errors for the non-matching combination $(8,7)$ are between the matching combinations $(7,7)$ and $(8,8)$ as one should expect.

\section{Conclusion}

A non-conforming finite element method for non-matching grids in three dimensions was described and implemented. Our numerical results for a model problem clearly demonstrate that the technique performs as well as the standard conforming finite element method in higher dimensions. 


\section{References}

F. B. Belgacem. The mortar finite element method with lagrange multipliers. Numer. Math., 84:173-197, 1999.

F. B. Belgacem, L. Chilton, and P. Seshaiyer. The $h p$-mortar finite-element method for mixed elasticity and stokes problems. Comp. Math. Appl., 46: 35-56, 2003.

F. B. Belgacem and Y. Maday. The mortar element method for three dimensional finite elements. RAIRO Math. Mod. Numer. Anal., 31:289-302, 1997.

F. B. Belgacem, P. Seshaiyer, and M. Suri. Optimal convergence rates of $h p$ mortar finite element methods for second-order elliptic problems. RAIRO Math. Mod. Numer. Anal., 34:591-608, 2000.

C. Bernardi, Y. Maday, and A. Patera. Domain decomposition by the mortar finite element method. Asymptotic and Numerical Methods for PDEs with Critical Parameters, 384:269-286, 1993.

D. Braess and W. Dahmen. Stability estimates of the mortar finite element method for 3dimensional problems. EastWest J. Numer. Math., 6:249-263, 1998.

D. Braess, M. Dryja, and W. Hackbusch. Multigrid method for nonconforming finite element discretization with application to non-matching grids. Comput., 63:1-25, 1999.

W. D. D. Braess and C. Wieners. A multigrid algorithm for the mortar finite element method. SIAM J. Numer. Anal., 37:48-69, 2000.

R. Ewing, R. Lazarov, T. Lin, and Y. Lin. Mortar finite volume element methods of second order elliptic problems. East-West J. Numer. Math., 8: 93-110, 2000.

R. E. Ewing and R. D. Lazarov. Superconvergence of the mixed finite element approximations of parabolic problems using rectangular finite elements. East-West J. Numer. Math., 1:199-212, 1993.

C. Kim, R. Lazarov, J. Pasciak, and P. Vassilevski. Multiplier spaces for the mortar finite element method in three dimensions. SIAM J. Numer. Anal., 39:519-538, 2001.

P. Seshaiyer. Stability and convergence of nonconforming $h p$ finite-element methods. Comp. Math. Appl., 46:165-182, 2003.

P. Seshaiyer and M. Suri. Convergence results for non-conforming $h p$ methods. Contemp. Math., 218:453-459, 1998.

P. Seshaiyer and M. Suri. $h p$ submeshing via non-conforming finite element methods. Comp. Meth. Appl. Mech. Engrg., 189:1011-1030, 2000a.

P. Seshaiyer and M. Suri. Uniform $h p$ convergence results for the mortar finite element method. Math. Comp., 69:521-546, 2000b.

B. I. Wohlmuth. A mortar finite elment method using dual spaces for the lagrange multiplier. SIAM J. Numer. Anal., 38:989-1012, 2000. 